\title{
THE MEASUREMENT OF THE INDUCTANCES OF RESISTANCE COILS
}

\author{
By Frederick W. Grover and Harvey L. Curtis
}

\section{CONTENTS.}

I. INTRODUCTION.

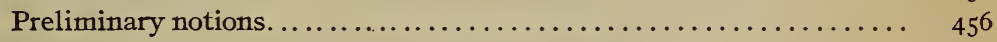

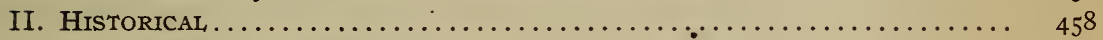

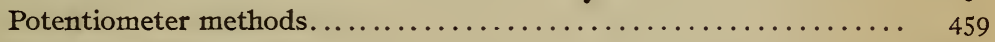

Resonance methods................................ 459

Dynamometer methods.............................. 459

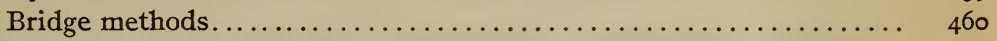

III. MEASUREMENT of The Inductance of CoIls of Low Resistance... 46

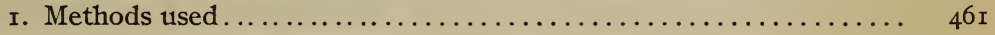

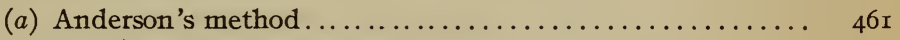

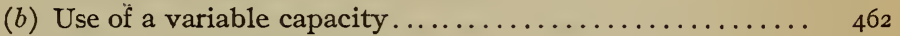

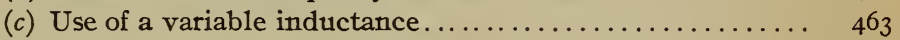

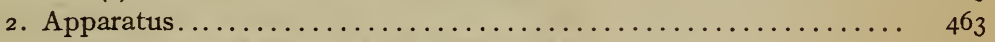

3. Calibration of the variable small resistance.............. 464

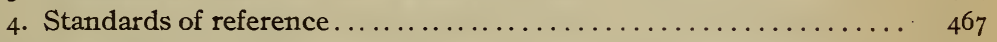

5. Experimental results........................... 468

IV. MEASUREMENT OF THE INDUCTANCE OF CoIls of High Resistance... 470

I. Effect of the capacity to earth on the standard............ 470

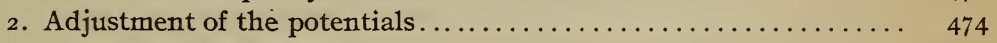

3. Experimental results........................... 479

4. Effect of the capacity to earth on the inductance to be measured... 479

V. Less PRecise Methods for High Resistance CoIL, ............. 48 I

I. Use of a bridge with unequal arms . . . . . . . .

2. Use of a standard in parallel with the unknown............. $48_{2}$

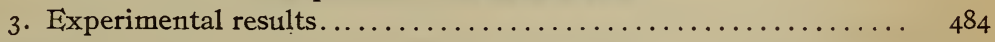

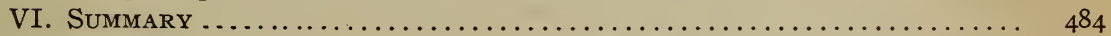




\section{INTRODUCTION}

In the choice of a bridge method for the measurement of a given inductance it is necessary to take into account not only the order of magnitude of the inductance in question but also the value of the associated resistance. That is, the determining factor is the ratio of the inductance to the resistance, or, as it is generally designated, the time constant of the coil, rather than the value of the inductance alone.

The use of Anderson's method for the precise measurement of the inductance of coils having a time constant of the order of 0.001 second or greater has been treated at length in a previous article of this Bulletin. ${ }^{1}$ Owing to the increasing precision demanded in electrical standardization, it is becoming more and more important to be able to measure the inductance of coils having time constants of much smaller value. For example, there may be cited the measurement of the small residual inductances of so-called noninductive resistance coils or of multipliers for voltmeter circuits. For such measurements the ordinary methods for the determination of inductance are more or less inapplicable or require extensive modification.

A good deal of time has accordingly been devoted to this subject at the Bureau of Standards, with the result that methods have been developed which are capable of giving results of a good degree of precision for time constants as small as $1 \mathrm{O}^{-7}$ second, such, for example, as that of a resistance of roo ohms having an inductance of a hundredth of a millihenry. It is hoped that a presentation of these methods and a discussion of the sources of error will be useful in stimulating interest in the subject and in calling attention to the errors which may arise from the use, without test, of apparatus supposed to be free from inductance.

\section{PRELIMINARY NOTIONS}

In addition to the effect of the inductance in causing the current in a given coil to lag behind the electromotive force impressed at its terminals, there has to be taken into account the influence of the capacity between the adjacent turns of wire and the capacity of the various parts of the coil with respect to the earth. These 
capacity effects give rise to a phase angle in the opposite direction to that occasioned by the inductance. The resulting phase angle will, therefore, be in one or the other direction, according as the effect of the inductance or that of the capacity preponderates.

It is convenient, in so far as its effect on the phase angle is concerned, to regard the capacity as equivalent to a negative inductance. For the ideal case of a capacity concentrated between the terminals of a coil, it is easy to show that the phase angle between current and impressed electromotive force is proportional to $L-C R^{2}$ (where $R, L$, and $C$ are, respectively, the resistance, inductance, and capacity of the coil), and the current lags or leads according as this quantity is positive or negative.

In the case of a simple bifilar winding, the capacity between the wires is uniformly distributed, and the resultant phase angle depends on the value of $L-\frac{\mathrm{I}}{3} C R^{2}$, where $C$ is the capacity which would be measured between the wires if they were entirely disconnected from one another.

In an actual coil the effect of the capacity between the wires and to earth can not in general be calculated. We may, however, speak of the effective inductance of the coil, meaning thereby that inductance which would, at the same frequency, produce the observed phase angle. According to this definition the effective inductance $L^{\prime}$ is connected with the measured phase angle $\phi$ (which may be positive or negative) by the equation $\tan \varphi=\frac{p L^{\prime}}{R}$, where $p=2 \pi$ times the frequency, $L^{\prime}$ being taken positive when the current lags behind the impressed electromotive force.

It is also often convenient, when the resistance is large, to regard the actual coil as replaced by an equivalent bifilar winding, having the same resistance, zero inductance, and a distributed capacity $C^{\prime}$ of such a value that the phase angle, calculated by the formula $\tan \phi=-p C^{\prime} R / 3$, is equal to the observed phase angle of the coil.

$$
56109^{\circ}-\mathrm{I} 2-2
$$


In the case of low-resistance coils, the measured phase angle is positive and it is easy to show that the capacity effect is negligible. Since, however, the change in phase angle, due to a given capacity, is, as shown above, proportional to the square of the resistance associated with it, it is easy to understand that in coils of high resistance the capacity becomes the predominating factor.

Hence, the problem of the measurement of the effective inductance of a I-ohm coil, having a time constant of $1 \mathrm{O}^{-8}$ second, is quite different from that of the determination of the effective inductance of a ro ooo-ohm coil having the same value of time constant. In the case of the I-ohm coil, the inductance of all lead wires must be allowed for or eliminated, and the mutual inductance of one bridge arm on another must be shown to be negligible, or a correction applied. In the measurement of the ro ooo-ohm coil, however, although the inductances of all ordinary lead wires and the mutually inductive effects of the various bridge arms may be regarded as of negligible importance, all capacities between the various parts of the bridge and to earth, even though no greater than a few millionths of a microfarad, may produce a measurable effect on the balance of the bridge.

The work, therefore, divides itself logically into two sections, which will be separately treated-first, methods for the measurement of the effective inductance of coils of small time constant and smiall resistance (I ohm or thereabouts); and, second, methods adapted to coils having small time constant, and resistances greater than about 1000 ohms. Coils of intermediate values of resistance may be treated by modifications (which will readily suggest themselves) of these methods for extreme values.

\section{HISTORICAL}

The various methods which have been previously employed for the measurement of the inductances of coils having small time constants, may be roughly grouped into four classes, which will be briefly reviewed below. However, the papers mentioned by no means exhaust the list which has been consulted. 
Potentiometer methods.-In 1909 Orlich ${ }^{2}$ published an electrometer method for determining the difference in inductance of two conductors, carrying the same current, the standard of reference being a rectangular bar, whose inductance was calculated from its dimensions. This method, which is especially well adapted to small resistances of high current-carrying capacity, and, in particular, those provided with potential terminals, gave an accuracy of about I per cent with coils having a resistance of $0.00 \mathrm{I}$ ohm and inductances of the order of $10 \mathrm{~cm}$.

Resonance methods. - Taylor ${ }^{3}$ measured very small inductances by a substitution method in a resonating circuit. Two parallel wires with a movable contact served as a standard, the inductance being calculated from the length and diameter of the wires and their distance apart. He confined himself entirely to coils of small resistances, an accuracy of a few per cent being obtained with time constants of about $1 \mathrm{O}^{-5}$ second.

Dynamometer methods. - A number of electrodynamometer methods have been devised for the measurement of small inductances, their essential differences lying, for the most part, in the means employed for compensating the change in phase produced by the insertion of the unknown coil into the circuit.

For example, in the method of Blondel ${ }^{4}$, who used a simple electrodynamometer and two phase currents, the inductance to be measured is made to depend on the value of a known inductance or a known resistance, or on both.

Martienssen ${ }^{5}$ employed a special electrodynamometer with a suspended metal cylinder as moving system, and restored the original adjustment of the circuits for zero torque on the cylinder (disturbed by the introduction of the unknown coil) by varying the resistance in the circuit of an auxiliary field coil. The inductance of the coil to be measured was thus made to depend on a known mutual inductance and a ratio of two resistances.

In none of these dynamometer. methods, however, does any

${ }^{2}$ Zs. für Instk., 29, p. 24I; 1909.

3 Phys. Rev., 19, p. 273; I904.

'Ecl. Elect., 21, p. I39; I899.

5 Ann. der Phys., 67, p. 95; 1899. 
high degree of accuracy seem to have been obtained with coils of very small time constant.

Bridge methods.-Essentially two bridge methods have been employed, namely, (a) Maxwell's method for the comparison of two inductances ${ }^{6}$, and (b) Maxwell's method for the comparison of an inductance with a capacity ${ }^{7}$ together with Anderson's modification $^{8}$ of the same. In most cases alternating current of frequencies ranging from 256 to 3000 cycles have been used, although La Rosa ${ }^{9}$ obtained a satisfactory sensibility using direct current.

Wien and Prerauer ${ }^{10}$, using the method for the comparison of two inductances, measured inductances as small as $500 \mathrm{~cm}$ by indirect comparison with an absolute standard of one millihenry, through auxiliary standards of intermediate values, obtaining an accuracy of about I per cent with time constants of about IO $^{-5}$ second.

In a low-inductance bridge such as was used by them, the simple relation derived by Maxwell no longer holds, but the inductances of all the arms of the bridge must be taken into account, and the method becomes increasingly inconvenient and subject to error as the time constant is smaller.

To obviate this difficulty, an important modification was introduced by Giebe ${ }^{11}$, who showed that, if relatively large inductances be inserted in the ratio arms of the bridge, the formula of Maxwell is subject to only a slight correction, which may be determined experimentally. Thus he was able to measure, with a good degree of accuracy, inductances of the order of $500 \mathrm{~cm}$ and with time constants as small as $10^{-8}$ second. Here, again, the inductance to be measured is made to depend on a standard, whose value can be calculated from its dimensions.

The method of comparing an inductance with a capacity has been used to measure the effective inductance of resistance coils of the order of Iooo ohms, by Taylor and Williams ${ }^{12}$ and by Brown. ${ }^{13}$

\footnotetext{
6 Elect. and Mag., vol. 2, art. 757.

7 Elect. and Mag., vol. 2, art. 778 .

${ }^{8}$ Phil. Mag., 31, p. 329: I89r.

9 Atti della R. Accad. dei Linc.; June 3, 1905.

${ }^{10}$ Wied. Ann., 53, p. 772; 1894.

11 Ann. der Phys., 24, p. 94I; 1907.

12 Phys. Rev., 26, p. 417; I908.

13 Phys. Rev., 29, p. 369; 1909.
} 
In both cases the unknown coil was compared, by substitution, with a standard of the same nominal resistance. The rooo-ohm standard, employed by Brown, was made by stretching two German silver wires parallel to one another, and at such a distance apart that the calculated inductance and capacity effects were balanced.

\section{MEASUREMENT OF THE INDUCTANCE OF COILS OF LOW RESISTANCE}

Attention has already been called the fact that the direct measurement of the inductance of coils of small resistance is rendered difficult by the necessity for knowing the inductances of all lead wires and auxiliary resistance coils and the mutual inductances between the various arms of the bridge. In the case of coils having relatively large time constants these difficulties may in part be avoided by the use of a method of difference. The bridge is successively balanced with the unknown coil in place and with the coil removed, the change in balance giving a measure of the required inductance. For the success of this method it is necessary to take into account the inductance of the resistance coils, which must be added to compensate for the resistance of the coil, as well as the inductance of the conductor used for closing the gap left by the coil on its removal.

The ideal method of procedure is to measure the unknown coil by a method of substitution, using a standard of exactly the same resistance as the unknown, but of such a form as to allow its inductance to be calculated from its dimensions, and in so far as was found possible the methods here employed were made to conform to this condition. The preparation of absolute standards for these measurements and the method of measuring the inductance of the small variable resistance used in balancing the bridge will be separately treated below.

\section{METHODS USED}

a. Anderson's method.-The standard having been inserted in the $Q$ arm of the bridge, Fig. I, the bridge is balanced by alternately varying the resistance $r$ and the resistance $Q^{\prime}$ in the $Q$ arm, external to the standard. To obtain balance with a convenient value of $r$, it may be necessary to place a small auxiliary inductance 
in series with the standard. Balance having been obtained, the unknown is substituted for the standard, and, everything else being left untouched, the bridge is again balanced by varying $r$

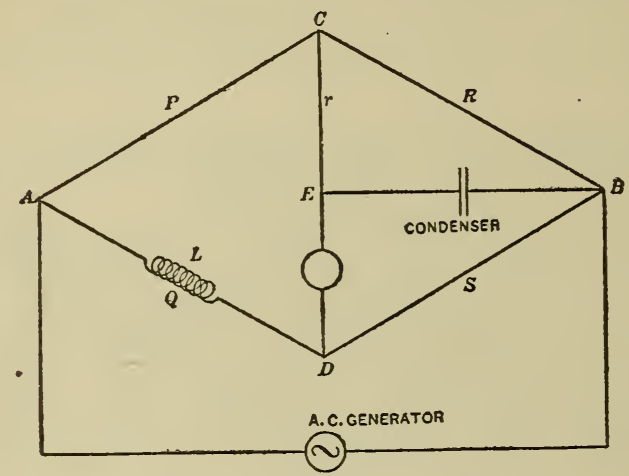

Fig. 1 -Anderson's Bridge

and $Q^{\prime}$. If the observed changes in $r$ and $Q^{\prime}$ be denoted, respectively, by $\Delta r$ and $\Delta Q$, and if $\Delta l$ be the change in inductance corresponding to $\Delta Q$, the difference in inductance, $\Delta L$, between the unknown and the standard is given by the relation

$$
\Delta L=2 C S . \Delta r-\Delta l
$$

Since $\Delta l$ is a correction, it should be made as small as possible, which is the reason for using a standard whose resistance is adjusted to be closely equal to that of the unknown. Care must, of course, be taken to take into account the algebraic signs of $\Delta r$ and $\Delta l$.

In order that $\Delta r$ may be as large as possible in any given case, $C$ and $S$ should be made small. Reducing $C$, however, tends to reduce the sensitiveness of the setting of the bridge. There is, however, rather a wide latitude allowable in the choice of the bridge constants.

b. Use of variable capacity.-For coils having a very small inductance, it is often advantageous to omit the resistance $r$ in the bridge and to obtain a balance by adjusting the capacity instead. This is a special modification of Maxwell's method for comparing an inductance with a capacity.

If $\Delta C$ denote the change in the capacity necessary to obtain a 
balance, when the unknown coil is substituted for the standard, the difference in inductance between the unknown and the standard is given by the equation

$$
\Delta L=P S \cdot \Delta C-\Delta l .
$$

The variation of $C$ is conveniently obtained by means of a calibrated variable air condenser.

In case the two above methods are combined, it is about as easy to obtain $\Delta L$ from the two values of $L$ as to use the formula for $\Delta L$. (See Example I below.)

c. Use of a variable inductance.-If a calibrated variable inductance of suitable range is at hand, it may be used, in series with the standard of reference, in one of the arms of a simple Maxwell's bridge for comparing two inductances. Then

$$
\Delta L=L_{o}-L_{1}-\Delta l
$$

where $L_{o}$ is the reading of the variable inductance with the standard in the bridge and $L_{1}$ the reading with the unknown.

For the calibration of the variable inductance one of the foregoing methods was used.

\section{APPARATUS}

. The current for the measurements at low frequencies was taken from a one-half kilowatt generator, driven by a motor which was supplied from a storage battery. The frequency of the current was about roo cycles per second, the exact value being determined by the natural period of the Ruben's vibration galvanometer, used to determine the point of balance of the bridge. The impedance of the galvanometer being considerably larger than the impedance of the other arms of the bridge, it was found advantageous to use a step-up transformer in the galvanometer circuit. With a $3: 1$ ratio on a 600-watt transformer the sensitiveness of the bridge setting was a little more than doubled.

The high frequencies, 200 to 3000 cycles, were obtained from an alternator with two stationary armatures and revolving fields, capable of giving 4.5 amperes at I Io volts at frequencies of 600 or 3000 cycles per second. By varying the speed of the driving motor a range of frequencies from 200 to 3000 cycles may be 
obtained. In the absence of a vibration galvanometer adapted to these frequencies, a low-resistance telephone served as indicating instrument in balancing the bridge, a procedure which was allowable in the present instance, since the conditions for balance, in the bridge methods used, are sensibly independent of the frequency.

Inasmuch as the change in the total impedance due to a given small change of inductance becomes more important with increasing frequency, the sensibility of the bridge should be greater at the higher frequencies than at the lower. On the other hand, the sensibility of the ear begins to decrease for sounds of frequencies much above Iooo per second, and, in addition, the natural periods of vibration of the diaphragm of the telephone have an appreciable effect on the position of the observed frequency for maximum sensitiveness. Thus, for example, with one of the telephones used, the maximum sensitiveness of the bridge was reached with a frequency of about I500 and had fallen off greatly at 3000 . With the other telephone, on the contrary, the maximum sensitiveness probably lies somewhat above 3000 cycles per second. In both cases the telephone became silent when the balance point of the bridge was reached, no trouble being experienced from higher harmonics. This does not, however, necessarily point to any very high degree of purity of the emf wave employed, since the effect of the reduced sensibility of the ear for these very high frequencies is to largely diminish the effective amplitude of the harmonics relatively to that of the fundamental.

\section{CALIBRATION OF THE SMALL VARIABLE RESISTANCE}

In the ideal substitution method the resistances of the unknown and the standard would be exactly equal, and it would be merely necessary to provide means in the bridge for varying the inductance without any accompanying change in the resistance. Since, however, tiie desired degree of approximation of the resistances of unknown and standard is often difficult of attainment, it is desirable to devise means for changing the resistance without, at the same time, appreciably changing the inductance, in order that the 
correction $\Delta l$ in equations $\mathrm{r}, 2$, and 3 may be kept as small as possible. The problem of obtaining change in inductance with a constant resistance has been successfully solved in a number of existing forms of variable inductances. The reverse problem of obtaining variation of resistance with a constant inductance is not so easy of solution.

An obvious method for doing this is to prepare resistances of simple forms, having small but calculable inductances, one of the units being provided with a sliding contact to allow of a continuous variation of resistance in balancing the bridge. It is, however, difficult, if not impossible, to construct a slide wire with a contact resisțance sufficiently constant to be satisfactorily employed in one arm of a low-resistance bridge. Further, the variation of the resistance in steps, with a negligible resultant change in the inductance, may be carried out more simply as follows:

Links are prepared of two metals of widely different specific conductivities (for example, copper and manganin), the wires being drawn through the same die so as to have the same diameter, and cut and bent so as to have, as closely as possible, the same form and dimensions. It is easy to so determine the length that the resistances of the copper and the manganin links shall differ by some convenient value, such as o.I ohm. Mercury cups are arranged so that any link may be placed in the circuit in the same, perfectly definite, position as any other, and with the ends of the wire dipping into the mercury just sufficiently to make contact. If now, for example, one of the copper links be replaced by a manganin link, the resistance in circuit is increased by o.I ohm, but since one link has exactly the same dimensions and occupies exactly the same position in the circuit as the other, the change of inductance, even with a considerable skin effect, is entirely negligible.

To provide the necessary means for obtaining a continuous variation of the resistance over a range of one-tenth ohm, a slight modification of the form of variable mercury resistance ${ }^{14}$ designed 
by Dr. F. Wenner and Mr. J. H. Dellinger of the Bureau of Standards was employed. Two straight glass tubes, one having approximately ten times the cross section of the other, were joined together as a $U$-tube and mounted vertically. The system was then filled with mercury up to the copper terminals, which extended into small glass cups blown on the end of each tube, to prevent any overflow of the mercury. To obtain the variation of resistance two copper wires are selected, of such diameter that each shall closely fill the cross section of its respective tube. Owing to the greatly superior conductivity of the copper, it effectively short circuits that portion of the mercury column into which it dips. The larger tube serves for the finer adjustment of the resistance.

This form of variable resistance has shown itself eminently satisfactory for the present purpose. Whereas, with a carefully constructed slide-wire resistance in series with a total resistance of I ohm in the bridge arm, the fluctuations of the current were so large and erratic that no close settings of the bridge could be made, the mercury variable was, on the contrary, free from all fluctuations of resistance as great as one hundred-thousandth of an ohm, only a small steady drift of resistance, due to temperature changes, being noted.

The mercury variable offers a further valuable advantage, in that its inductance changes only very slightly as the resistance is varied. If the copper wires could be made exactly to fill the cross section of their tubes, the inductance would, with currents of low frequency, be sensibly independent of the position of the wires. Since, however, this condition is only approximately realized, it is necessary to calibrate the instrument. This was accomplished by means of copper and manganin links of suitable lengths as described above. As a result of this calibration it was found that the change of inductance is practically linear, the changes in inductance corresponding to the entire ranges of the fine and coarse adjustments, being only about 12 and $7 \times 1 \mathrm{IO}^{-9}$ henrys, respectively. Since it is often unnecessary to make any considerable changes in the fine adjustment when balancing the bridge, the more considerable source of variation of the inductance may usually be avoided. 
It may be noted in passing that the use of such a variable is not confined to currents of low frequencies, since it is pcssible by calculation to correct the calibration with all the accuracy necessary to take into account the skin effect.

\section{STANDARDS OF REFERENCE}

The forms of standards for use in the substitution method which most readily suggest themselves are parallel wires, rectangles, and circles.

The inductance of two parallel wires of permeability $\mu$, radius of wire $\rho$, length $l$ (of a single wire) and distance apart $d$, is given with sufficient accuracy by the formula

$$
L=4 l\left[\log _{e} \frac{d}{\rho}+\frac{\mu}{4}-\frac{d}{l}\right]
$$

provided $l$ is greater than ro $d$ and $d$ is greater than Io $\rho$.

If the wires are close together or, in any case, if the resistance is large, it may be necessary to take into account their capacity. The effective inductance is given by

$$
L^{\prime}=L-\frac{C R^{2}}{3}
$$

where $R$ is the resistance of the wires and $C$ is the capacity which would be measured between the wires if they were disconnected from one another. The calculation of $C$ is treated later.

For circles of wire of circular cross section

$$
L=4 \pi a\left[\left(\mathrm{I}+\frac{\rho^{2}}{8 a^{2}}\right) \log _{e} \frac{8 a}{\rho}+\frac{\rho^{2}}{24 a^{2}}-\mathrm{I} .75\right]
$$

where $a$ is the radius of the circle, and $\rho$ is the radius of the cross section.

The principal source of uncertainty in the use of such standards of reference lies in the difficulty of estimating the inductance and mutual inductive effects at the terminals. The use of large binding posts is to be avoided. That no appreciable inconsistency resulted from these causes in the standards here used is shown by the following examples. 


\section{EXPERIMENTAL RESULTS}

EXAMPLE 1

Two standards of I-ohm resistance were prepared, both of manganin wire $0.4 \mathrm{~mm}$ in diameter, the wire in one being arranged in a loop with the two legs I cm apart, while in the other it was bent into a circle of $5.80 \mathrm{~cm}$ radius with a gap of $1.8 \mathrm{~cm}$ between the ends. Each standard was provided with binding posts, in one case set perpendicular to the plane of the parallel wires, and in the other perpendicular to the plane of the circle. The self inductance of the binding posts was calculated and the mutual inductance with respect to the wire was assumed to be negligible.

The inductances were calculated, giving the following values:

Parallel wires.

279

8 End

4 Binding posts

29 I cm
Circle.

\section{7}

- 22 Gap correction

4 Binding posts

$419 \mathrm{~cm}$.

The calculated difference was therefore $128 \mathrm{~cm}$. The difference actually measured by a combination of methods I and 2, above, was $128 \mathrm{~cm}$, thus agreeing to the nearest $\mathrm{cm}$. The details of this measurement are given below.

Referring to Fig. I, the $P$ and $R$ arms each included a I-ohm sealed resistance standard. In the $S$ arm, in addition to a I-ohm standard, was placed a coil consisting of three or four turns of copper wire wound to a radius of about $4 \mathrm{~cm}$. The $Q$ arm was made up of the unknown (or standard) to which was added the variable mercury resistance already described. The I-ohm standards were hung between the mercury cups of a bridge designed for precision resistance measurements. All other connections were made to the binding posts of this bridge, and thus was avoided any indefiniteness in the relative positions of the parts of the bridge, a very necessary precaution with such small inductances. The small inductance inserted in the $S$ arm was to insure obtaining a balance of the bridge. A small resistance was used in $r$ for convenience, and for the capacity a $\mathrm{O} . \mathrm{I}-\mu \mathrm{f}$ condenser, shunted by a $0.005^{-\mu \mathrm{f}}$ variable air condenser was employed. 
The latter could be set to about I degree of the scale which corresponds to about $22 \times 10^{-12}$ microfarad, or 2 parts in 10000 of the whole capacity. The measured value of the resistance $S$ was r.056 ohms. The remaining data are as follows:

\begin{tabular}{|c|c|c|c|c|c|}
\hline & $\mathbf{r}$ & C & $\operatorname{CS}(2 r+P)$ & Correctèd & $\mathbf{L}$ \\
\hline & ohms & $\mu \mathrm{f}$ & $\mathrm{cm}$ & $\mathrm{cm}$ & $\mathrm{cm}$ \\
\hline Circle.. & 3.1 & 0.10217 & 778 & 778 & (419) \\
\hline Parallel wires. & 3.7 & 0.10178 & 905 & 906 & 291 \\
\hline Coil 19a... & 4.9 & 0.10217 & 1167 & 1167 & 29 \\
\hline Coil 20a.. & 4.9 & 0.10180 & 1161 & 1161 & 35 \\
\hline
\end{tabular}

For the order of accuracy aimed at ( $\mathrm{I}$ in IOoo) the change in $r$ could be taken as equal to the difference in the nominal values. The values in the fifth column are corrected for the inductance of the mercury resistance. The last column gives the absolute values of the inductances, assuming for the circular standard the value calculated above. Coils r $9 \mathrm{a}$ and $20 \mathrm{a}$ are specially wound coils.

(NOTE.-The capacity was connected in this bridge not, as in Fig. I, to the junction of $R$ and $S$, but to the junction of $P$ and $Q$. The reading of the bridge increases, therefore, for a decrease in the inductance in $Q$.)

EXAMPLE 2

The accuracy of the methods was also tested for resistances of larger values. For this purpose parallel wire standards of manganin wire, stretched on vulcanite spacing blocks attached to a long board, were prepared, of such values as to allow a step up from lower values to higher, and the observed differences in inductance could thus be compared with the calculated.

It was found convenient to employ method 3 in the measurements, making use of a previously calibrated variable inductance of about I2-microhenrys range. The other three arms of the bridge were made up of resistance standards hung in mercury cups, and to take care of the small differences in resistance, the tenth ohms of copper and manganin links and the variable mercury resistance were used. Care was taken, when using two or more parallel wire standards, to place them perpendicular to one another, to avoid effects of mutual induction. 
The following table gives an idea of the consistency of the results obtained, and some estimate can be made as to how thoroughly constant errors have been eliminated.

\begin{tabular}{|c|c|c|}
\hline Coils compared & $\begin{array}{l}\text { Observed } \\
\text { difference }\end{array}$ & $\begin{array}{l}\text { Calculated } \\
\text { difference }\end{array}$ \\
\hline $100_{1}-100_{2}$ & $\begin{array}{c}\text { Microhenrys } \\
0.16\end{array}$ & $\begin{array}{c}\text { Microhenrys } \\
0.17\end{array}$ \\
\hline $100_{1}-100_{3}$ & 0.16 & 0.17 \\
\hline$\left(200+100_{3}\right)-300$ & 2.79 & 2.71 \\
\hline$\left(100_{1}+100_{2}+100_{3}\right)-300$ & 7.83 & 8.08 \\
\hline$(300+200)-500$ & 3.67 & 3.52 \\
\hline$(300+200)-500_{2}$ & 4.02 & 3.59 \\
\hline$\left(500+300+100_{2}+100_{3}\right)-1000$ & 11.4 & 11.5 \\
\hline$\left(500_{1}+500_{2}\right)-1000$ & 2.92 & 3.04 \\
\hline
\end{tabular}

\section{MEASUREMENT OF THE INDUCTANCE OF COILS OF HIGH RESISTANCE}

In the previous section it has been shown that, over a wide range of resistances, the simple substitution method may be depended upon to give reliable values of the inductance. However, in the measurement of coils whose resistance is of the order of 1000 ohms, or greater, where a small capacity can produce an appreciable change of the phase angle, special care must be taken to avoid serious errors. The effect of the capacity between the windings has already been discussed and has been shown to be proportional to the square of the resistance around which the capacity is shunted. There remains yet to be considered the action of the capacity between the various parts of the coil and the earth, and it will be shown that this effect, which is negligible in the case of low resistances, may be important with coils of no greater resistance than a few thousand ohms.

It is convenient to consider separately in this connection (a) the earth capacity of the standard and (b) that of the coil to be measured.

\section{EFFECT OF THE CAPACITY TO EARTH ON THE STANDARD}

If the two parallel wires represented in section in Fig. 2 be brought, respectively, to potentials $v_{1}$ and $v_{2}$, the quantities of 
electricity $q_{1}$ and $q_{2}$ on the wires will be given by

$$
\begin{aligned}
& q_{1}=c_{1} v_{1}+c_{12}\left(v_{1}-v_{2}\right) \\
& q_{2}=c_{2} v_{2}-c_{12}\left(v_{1}-v_{2}\right)
\end{aligned}
$$

where $c_{1}, c_{2}$, and $c_{12}$ are positive constants depending upon the configuration of the system. These are the classical Maxwell

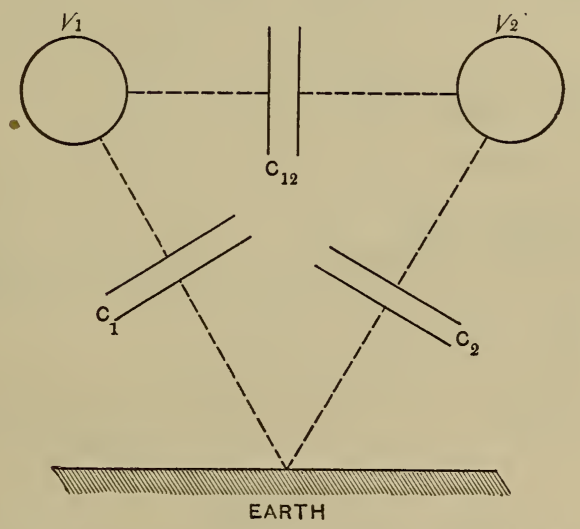

Fig. 2.-Capacities of Two Parallel Wires Above the Earth

equations, for a system of charged conductors, put into a modified and more convenient form. ${ }^{15}$ According to these equations, we may regard the quantity of electricity on I as composed of two parts, viz, a displacement between the earth and $I$, and a displacement between 2 and I. Similarly, the quantity $q_{2}$ on 2 is the resultant of a displacement between the earth and 2 and a displacement between I and 2. That is, if we consider the lines of electrostatic force, then $c_{1} v_{1}, c_{2} v_{2}$ and $c_{1}\left(v_{1}-v_{2}\right)$ are respectively proportional to the numbers of lines which join I and the earth, 2 and the earth and I and 2. The system I, 2, earth, is therefore equivalent to a network of three simple capacities arranged as in Fig. 2.

If, instead of two wires, we consider the case of two plates whose distance apart is small compared with their dimensions, as in an ordinary condenser, the coefficient $c_{12}$ is very large compared with $c_{1}$ and $c_{2}$, and we may, without sensible error, speak of the capacity of the condenser without specifying the surroundings of

15 Orlich: Kapazität und Induktivität (Friedrich Vieweg u. Sohn, Braunschweig), pp. 20-22. 
the latter. Where, however, $c_{1}$ and $c_{2}$ become relatively more important as in the case of the wires it is evident that the quantities of electricity $q_{1}$ and $q_{2}$ will in general be different and will depend on the potentials $v_{1}$ and $v_{2}$. If an alternating potential difference be impressed upon the two wires, the charging current flowing on to one wire, at any instant, will not, in general, be equal to that flowing away from the other, since the displacement current between one wire and the earth will not be equal to that flowing between the other wire and the earth.

It is evident, therefore, that one can not speak of the capacity of the wires in the usual sense. The phase difference of the parallel wire standard depends not only on the size of the wires and their distance apart, but also on their absolute potentials, and can not usually be simply calculated.

However, in the important special case that $q_{1}=-q_{2}$ the general equations above take the following simple forms:

$$
\begin{gathered}
c_{1} v_{1}=-c_{2} v_{2} \\
C=\frac{q}{v_{1}-v_{2}}=c_{12}+\frac{c_{1} c_{2}}{c_{1}+c_{2}}
\end{gathered}
$$

The quantity $C$ here is the capacity, in the usual sense, between the two wires, and is seen to be equal to the joint capacity of the condenser $c_{12}$ joined in parallel with two condensers $c_{1}$ and $c_{2}$ in series.

In the case of two equal parallel wires $c_{1}=c_{2}$ and the equations become

$$
v_{1}=-v_{2} \text { and } C=c_{12}+\frac{c_{1}}{2}
$$

Therefore, if the absolute potentials of the two wires be so adjusted that, at every moment, the potential of one is just as far above the earth potential as the other is below it, not only will the quantities of electricity on the two wires be equal and of opposite sign, but they will be proportional to the potential difference between the wires. That is, the displacement current is the same as would flow in a single condenser (capacity to earth negligible) having a capacity $C=c_{12}+\frac{c_{1}}{2}$, subjected to the same voltage. 
This capacity $C$ is what is ordinarily designated (without, however, a clear statement of the conditions to be fulfilled by the potentials) as the "capacity" of the system. Orlich, however, whose method of treatment has been followed here, very clearly states the conditions (loc. cit., p. 26) and recommends for the capacity $C$ the name "Betriebskapazität" or "working capacity," $a^{\prime}$ term which will be employed below.

The working capacity of two parallel wires arranged at equal distances from the earth is ${ }^{16}$

$$
C=\frac{k l}{4 \log _{e} \frac{2 h}{\rho}-2 \log _{\mathrm{e}}\left(\mathrm{I}+\frac{4 h^{2}}{d^{2}}\right)} \text { electrostatic units }
$$

where

$k=$ dielectric constant of the medium

$l=$ length of each wire

$\rho=$ radius of wire

$d=$ distance between the centers of the wires

$h=$ height of the wires above the earth.

This may be thrown into the form

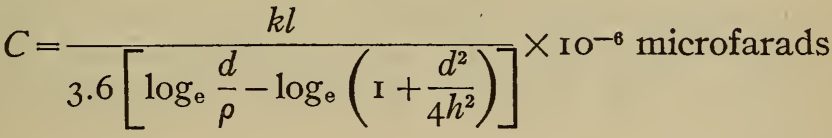

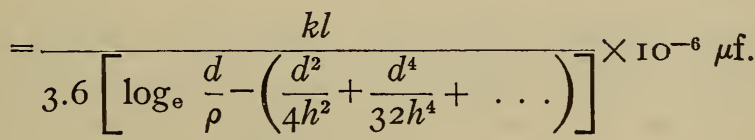

The correction terms in $\frac{d}{h}$ are, however, negligible in most practical cases. For example, in the case of two wires for which $l=\mathrm{I} 300 \mathrm{~cm}, d=\mathrm{IO} \mathrm{cm}, \rho=0.0025 \mathrm{~cm}$; the difference of the capacity as calculated with and without the terms in $\frac{d}{h}$ for a value of $h$ as small as Io $\mathrm{cm}$, was only 3 per cent; and with $h=100 \mathrm{~cm}$ the difference was smaller than the errors of observation.

The working capacity of a parallel wire standard can be made, 
therefore, perfectly definite and can be accurately calculated by equation (II); provided, of course, that the absolute potentials of the wires satisfy the condition $v_{1}=-v_{2}$, as discussed above.

This condition is, however, of prime importance, as was shown by experiment. For example, with an equal arm bridge, insulated from the earth, the observed value of the capacity of the parallel wires, whose dimensions are given above, was twice as great as that calculated. With small resistances this source of error is not important. On the other hand, with the 5000-ohm standard, which was made by joining the further ends of these wires, the phase difference depends almost entirely on the capacity. In order, therefore, to use the calculated constants of the standard, means must be provided for the adjustment of its potential so as to satisfy the condition $v_{1}=-v_{2}$, or, what is equivalent, to adjust the potential of the middle of the coil to zero potential.

\section{ADJUSTMENT OF THE POTENTIALS}

Fig. 3 represents a bridge to one arm of which have been connected two parallel wires $A$ and $B$. In accordance with Fig. 2 , we may represent their impedances due to their capacities to earth by $\alpha$ and $\beta$, their connections being drawn in dotted lines to distinguish them from the actual conductors of the bridge. The capacity $c_{12}$, Fig. 2 , is not indicated, but is included in the impedance $R$.

The desired condition which the potentials $v_{1}$ and $v_{2}$ of $A$ and $B$ are to satisfy is that $v_{1}$ shall be as much above earth potential as $v_{2}$ is below it, or vice versa. The supply voltage may be taken from a transformer or from a rheostat $B$ placed across the terminals of a well-insulated generator. In either case, if the entire system is insulated from the earth, the center will be close to earth potential. The potentials of $A$ and $B$ will not, therefore, normally fulfill the desired condition, and means must be provided to arbitrarily ground the supply at the correct point. In figure 3 this is done by means of a variable contact $D$, grounded through an impedance $M$. The total voltage $E$ of the supply is thus divided into two parts $K E$ and $(K-\mathrm{I}) E$, one being above and the other below earth potential. We will next consider what value $K$ must have in order that the potentials of the wires $A$ and $B$ 
shall have values symmetrically above and below the earth potential.

Indicate the impedances of the different arms by the symbols used in Fig. 3 and designate by $x, y, z, u$, and $v$ the currents in

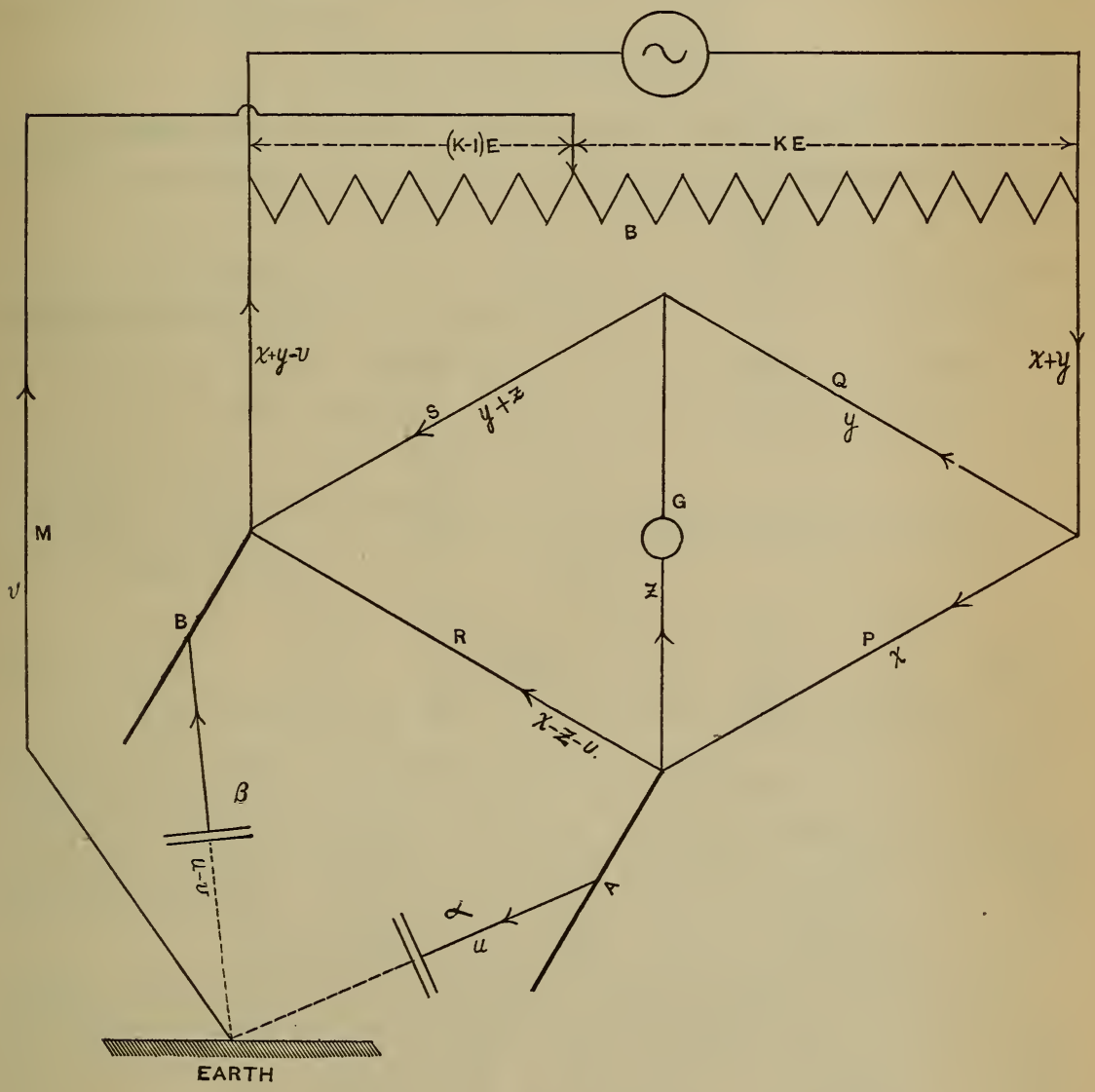

Fig. 3.-Special Bridge in which the Impedances and Currents are Indicated

the impedances $P, Q, G, L$, and $M$, respectively. Then by Kirchhoff's first law, the currents through the various impedances will be as follows:

$P, Q, \quad R, \quad S, \quad G, \alpha \quad \beta \quad K B, \quad(K-\mathrm{I}) B, \quad M$ $x, y,(x-z-u),(y+z), z, u,(u-v),(x+y),(x+y-v), \quad v$. 
Applying Kirchhoff's second law to five of the closed circuits of the network, the following equations are deduced:

$$
\begin{aligned}
P x-Q y+G z & =0 \\
R(x-z-u)-S(y+z)-G z & =0 \\
R(x-z-u)-\beta(u-v)-\alpha u & =0 \\
K B(x+y)+P x+\alpha u+M v & =K E \\
K B(x+y)+Q y+S(y+z)+(K-\mathrm{I}) B(x+y-v) & =\mathrm{E} .
\end{aligned}
$$

The general condition which must be satisfied that the current in the galvanometer arm may be zero $(z=0)$ is as follows:

or

$$
\left|\begin{array}{ccccc}
P & -Q & o & o & o \\
R & -S & -R & o & o \\
R & o & -(R+\alpha+\beta) & 0 & \beta \\
K B+P & K B & \alpha & K E & M \\
B & B+Q+S & o & E & (K-I) B
\end{array}\right|=0
$$

$$
\begin{aligned}
& {[K(K-I) B E-E M]\left|\begin{array}{ccc}
P & -Q & o \\
R & -S & -R \\
R & o & -(R+\alpha+\beta)
\end{array}\right|} \\
& +E \beta\left|\begin{array}{ccr}
P & -Q & o \\
R & -S & -R \\
K B+P & K B & \alpha
\end{array}\right|-K E \beta\left|\begin{array}{ccc}
P & -Q & 0 \\
R & -S & -R \\
B & (B+Q+S) & o
\end{array}\right|=0
\end{aligned}
$$

For correct adjustment of the potentials, there will be no resultant displacement from the wires $A$ and $B$ to earth; that is, the displacement current $u$ from $A$ to earth is equal to the displacement current $(u-v)$ from the earth to $B$. This means that $v$ must equal zero, or there will be no current in $M$.

Therefore, for simultaneous balance of the bridge and correct adjustment of the potentials of $A$ and $B$ two conditions must be fulfilled, namely, $z=0$ and $v=o$. The desired value of $K$ may be obtained from the solution of these two simultaneous equations. The work may, however, be simplified from the following consideration:

When the current $v$ is zero, the potential difference on the impedance $M$ must also be zero, and since that portion of the current $z$, which is due to the presence of $M$, must vanish when the potential difference on $M$ is zero, the coefficient of $M$ in equa- 
tion (13) must become zero when $v=0$. That is-

$$
\left|\begin{array}{crc}
P & -Q & o \\
R & -S & -R \\
R & 0 & -(R+\alpha+\beta)
\end{array}\right|=0
$$

which is equivalent to

$$
P R S+(\alpha+\beta)(P S-R Q)=0
$$

The remaining two determinants in (I 3 ) reduce to the simple relation

$$
P Q R-K P R(Q+S)-\alpha(P S-R Q)=0
$$

which, on elimination of $(P S-R Q)$ by means of the preceding equation, gives finally

$$
K=\frac{Q+\frac{\alpha}{\alpha+\beta} S}{Q+S}
$$

or for two wires of the same dimensions, since $\alpha=\beta$

$$
K=\frac{Q+\frac{S}{2}}{Q+S}
$$

To check the work we have also expanded the determinant for $v$ and shown that if the conditions (I4) and (I5) are satisfied it reduces identically to zero.

The preceding discussion leads at once to a simple method for adjusting the potentials of the wires $A$ and $B$ (see Fig. 4). With wires of equal size, which is the only case with which we are concerned, the adjustment of the potentials of $A$ and $B$ will be correctly attained if the potential of the middle point $W$ of the resistance $R^{\prime}$ be made zero. The supply voltage to the bridge is taken from the terminals of a rheostat $B$, on which is a movable contact $D$, permanently connected to earth through the wire $F$. The key $N$ being open, the bridge is balanced as usual. Then, with $N$ closed, the position of the contact $D$ is changed until no current flows in the telephone $T$. The balance of the bridge will usually be somewhat disturbed by the latter adjustment, and should be restored and the position of $D$ again adjusted. The balance of the bridge 
is not appreciably affected by a small lack of adjustment (I per cant) of the potential of $W$, so that this second approximation should be ample. Nevertheless, the adjustment of $D$ having been accomplished, the circuit through $T$ should be left open while the measurements of inductance are being made, since any small current in $T$, due to imperfect adjustment of the potentials, has to flow in part through the galvanometer arm.

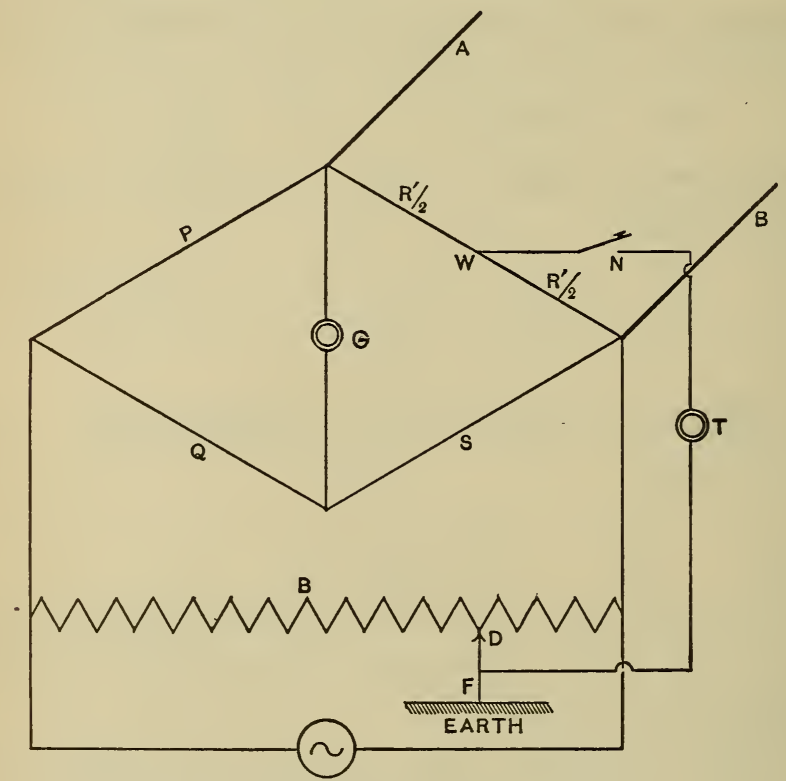

Fig. 4.-Method of Adjusting the Potentials

It should be noted that, in the analytical discussion of the bridge, it was tacitly assumed that the time constant of every part of the impedance $B$ is the same, so that the phase angle of the portions $K B$ and $(K-I) B$ are the same. Since this condition will rarely be exactly realized in practice, it will usually be necessary to insert a small variable inductance in $B$, at one or the other end of the circuit, in order to bring the telephone $T$ to silence. Usually, however, the adjustment of the potentials can be made with sufficient accuracy by setting $D$ to give a minimum sound in the telephone, and the inductance may be dispensed with. 


\section{EXPERIMENTAL RESULTS}

As a check on the sufficiency of the adjustment of the potentials by the method just described, measurements were made on a 5000-ohm parallel wire standard of $0.05-\mathrm{mm}$ manganin wire. The bridge having been balanced with the wires $10 \mathrm{~cm}$ apart, this distance was successively reduced to $5 \mathrm{~cm}$ and $2 \mathrm{~cm}$, and the change in inductance noted. In every case the observed difference agreed well with the computed, as the following table will show:

\begin{tabular}{c|c|c}
\hline \multirow{2}{*}{$\begin{array}{c}\text { Distances between the wires in } \\
\text { centimeters }\end{array}$} & \multicolumn{2}{|c}{ Difference of Inductance in microhenrys } \\
\cline { 2 - 3 } & Observed & Computed \\
\hline $10-5$ & 49 & 49 \\
$10-2$ & 114 & 110 \\
\hline
\end{tabular}

\section{EFFECT OF EARTH CAPACITY ON INDUCTANCE TO BE MEASURED}

It has been shown in the preceding sections that if the potentials of a parallel wire standard forming one of the arms of an alternating current bridge be so adjusted that the potential of one wire is as far above earth potential as the other is below it, the capacity of the standard may be calculated. The inductance and capacity being both calculable, the phase angle of the standard is known, and the substitution method, whose application to coils of rooo ohms or less has already been considered, may also be used for higher resistances, at least up to ro ooo ohms.

It must be emphasized, however, that the phase angle of a coil thus measured is the value holding only for the case that its center is at earth potential. Unless the capacity of the coil to earth is negligible, the phase angle of the coil will vary with any deviation from this distribution of potential, and this effect may be very important. Evidently, for this reason, the capacity of the coil with respect to the earth should be kept as small as possible.

This has special application to the connection of resistance coils in series. If, for example, we know the effective inductance of each of two coils, the effective inductance of the two coils in series 
can not in general be taken as the sum of the individual values. For if the phase angle of each coil has been measured with the center of the coil at zero potential, this condition can no longer hold for the combination in series. If the phase angle of the coils in series be measured by the substitution method, the point of zero potential will be the junction of the two coils. A further source of error lies in the fact that the mutual capacity effect of two adjacent coils in a resistance box is often appreciable.

Campbell ${ }^{17}$ suggested that each coil, or group of coils, in a resistance box be surrounded by a metal shield, joined to some definite point of the circuit. This procedure undoubtedly gives a perfectly definite capacity of the coil with respect to its surroundings, although the actual values of the capacity are thereby increased and need to be determined with correspondingly increased percentage accuracy.

If such a shielded resistance box is always used under exactly the same conditions which held during calibration, it undoubtedly may be depended upon to give a perfectly definite and known phase angle. If, on the contrary, the resistances are to be used for a variety of purposes, it will not, in general, be possible to keep the point of zero potential always at the same place in the system, and although the earth capacities of the coils are still perfectly definite, the values of phase angle will no longer be those of the calibration. In fact, the error in the phase angle, due to a given variation in the position of zero potential, will usually be much larger than in the case of well-designed and well-mounted unshielded coils. Just as has been shown for parallel wires, the capacity of a coil with respect to its surroundings varies only slowly with change of position, except in the immediate vicinity of extended metallic surfaces or other coils carrying current, and the effect of such changes will be reduced by keeping the capacity of the coil small. By experiment we find that with coils of small capacity the effect of changes of capacity with the position of the coil are only appreciable for distances between the coil and other coils and conductors less than about $5 \mathrm{~cm}$. 
It is accordingly best, at least in the case of coils to be used for a variety of purposes, to make the capacity of the coils as small as possible, and to mount each so that it is at least several centimeters from any other coil. The phase angle of each coil and of each desired combination should be measured by the substitution method, and, in so far as is possible, in using the coils the position of zero potential should be adjusted to conform with that for which the phase angles were measured. With properly designed coils of small capacity, not too close to one another, all extended metal surfaces being avoided in the neighborhood of the coils, the errors in phase angle owing to unavoidable deviations from the ideal positions of the point of zero potential will not be appreciable except in work of high precision.

\section{LESS PRECISE METHODS FOR HIGH RESISTANCE COILS}

When the effective inductances of resistance coils of large value have to be measured, the tise of the substitution method is attended by the difficulty of preparing a standard. For example, each wire of the 5000-ohm parallel wire standard of $0.05-\mathrm{mm}$ manganin wire, used in the present work, was 13 meters long. It may often, therefore, be convenient, where the highest precision is not desired, as in the case of coils of large capacity, to use one of the following methods.

\section{USE OF BRIDGE WITH UNEQUAL ARIMS}

The unknown coil is joined in one of the arms of a Wheatstone bridge, for example $R$, Fig. 5. The other arms are composed of resistances at least io times smaller than $R$ and of known effective inductances. The potential of the middle point of $R$ having been adjusted to that of the earth, the bridge is balanced by varying the resistance and inductance in $R$ by means of an auxiliary resistance of known effective inductance and a calibrated variable inductance in series with the unknown.

The condition for balance is

$$
l+l^{\prime}{ }_{3}=\frac{S}{Q} l_{1}+\frac{P}{Q} l_{4}-\frac{R}{Q} l_{2}
$$

$l$ being the unknown inductance and $l_{3}^{\prime}$ the total inductance in the 
$R$ arm external to the unknown. This will usually be sensibly equal to the reading of the variable inductance, since the effective inductance of the auxiliary resistance coils will be relatively negligible.

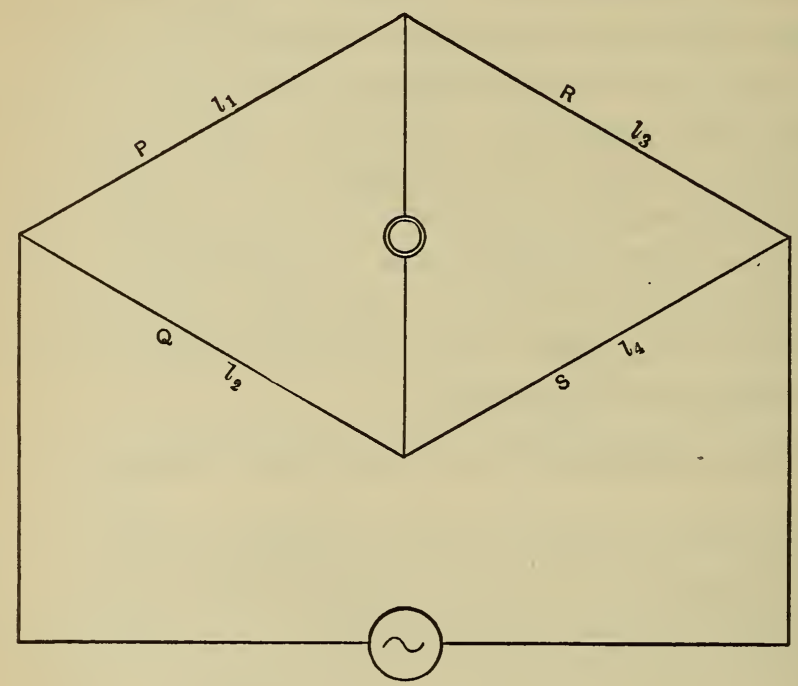

Fig. 5.-Wheatstone's Bridge

The coils $P, Q$, and $S$ being of relatively small value, their effective inductances will not be affected appreciably by their capacity to earth. It is, however, difficult, if not impossible, to avoid errors due to the earth capacity of the auxiliary apparatus in the $R$ arm in series with the unknown.

\section{USE OF A STANDARD IN PARALLEL WITH THE UNKNOWN}

In this method, see Fig. 6 , a coil of known resistance $R_{1}$ (much less than the unknown) and effective inductance $l_{1}$ is joined in series with a variable inductance $L_{2}$ of convenient value together with a variable resistance, the total resistance of this part of the arm being $R_{2}$. The bridge having been balanced by variation of $R_{2}$ and $L_{2}$ (a suitable fixed inductance $L_{4}$ being inserted in $S$ ) the unknown coil is joined as a shunt around the standard and the bridge is again balanced by varying $R_{2}$ and $L_{2}$. 
If $\Delta L_{2}$ be the observed change in the variable inductance when the unknown is introduced, we have, neglecting terms which are inappreciable at ordinary frequencies,

$$
l_{0}=-\left(\Delta L_{2}+\Delta l_{2}\right)\left(\frac{R_{1}+R_{0}}{R_{1}}\right)^{2}+l_{1}\left(\mathrm{I}+\frac{2 R_{0}}{R_{1}}\right)
$$

where $\Delta l_{2}$ is the change in the effective inductance of the resistance coils of $R_{2}$ when the unknown is introduced. Unfortunately, when

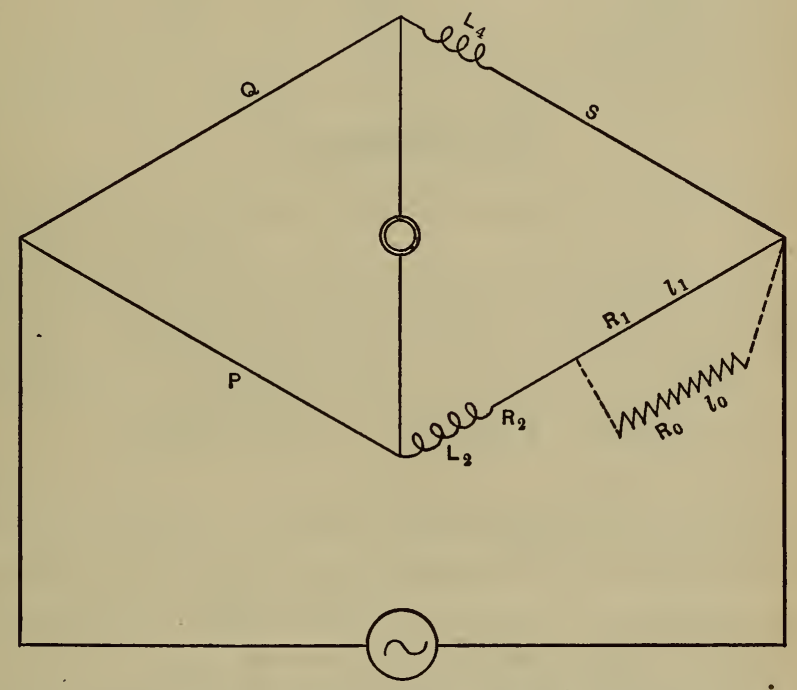

Fig. 6.-Diagram of Shunt Method for Measuring the Effective Inductance of a High Resistance

the effective inductance of the unknown is small, all of the terms are important, and $\Delta l_{2}$ and $l_{1}$ must be known with a good deal of accuracy. In this method, as in the preceding, the potential of the center of the unknown should be made zero, which may be done by so choosing the standard that connection may be made to its center.

In order that $\Delta L_{2}$ may be large, the resistances $R_{1}$ and $R_{0}$ should be of the same order of magnitude. This is, however, opposed to the essential advantage of the method-that the effective inductance of a large resistance is to be made to depend on that of a convenient standard of smaller value. 


\section{EXPERIMENTAL RESULTS}

The accuracy which may be easily attained by the use of these methods is shown in the following table:

Measured Effective Inductance in Millihenrys

\begin{tabular}{c|c|c|c}
\hline Coil & Method 1 & Method 2 & Substitution \\
\hline Multiplier 10 000 ohms & -1.4 & -0.5 & -1.8 \\
Wolff 2438 10 000 No. 1 & -111.3 & -112.3 & \\
Wolff 2438 10000 No. 2 & -113.3 & -114.1 & \\
Wolff 2438 Sum of 1 and 2 & -231.2 & -228.2 & \\
\hline
\end{tabular}

\section{SUMMARY}

I. In this paper is treated the measurement of the effective inductance of "noninductive" resistance coils and multipliers for use in alternating current bridges and in potential circuits where it is desired to know the phase angle of the circuit. The measurement of the inductances of very low resistance conductors, such as shunts with potential terminals, is not included.

2. The phase angle of a resistance coil is proportional to the resultant effects of its inductance and the capacities between the windings and between the various parts of the coil and the earth. The effective inductance is defined as that value of inductance, positive or negative, which would produce the observed phase angle.

3. In a low-resistance coil the effect of the inductance is most important; in a high-resistance coil the capacity is the determining factor, and the effect of the capacity of the coil to earth must be carefully taken into consideration. The method of treatment of the problem, therefore, varies according to the order of magnitude of the resistance of the coil.

4. The measurements with coils of low resistance are best made by means of a substitution method, using as a standard of reference a resistance of the same nominal value as the unknown, but of such a form that its inductance may be calculated from its dimensions. In this connection both parallel wires and circles were used.

5. To obtain small variations of resistance, with negligible change in inductance, wires of equal dimensions but of different conduc- 
tivities were substituted one for the other. A continuous variation of resistance over a small range was obtained by varying the effective length of a mercury column. The accompanying change of inductance was measured, but was practically negligible.

6. For resistances of the order of rooo ohms and greater the capacity of a parallel wire standard with respect to the earth becomes important. Attention is called to the fact that the usual formula for the capacity of such a standard applies only when the potential of one wire is as much above the earth potential as the other is below it. Failure to make this adjustment may easily give rise to capacity effects twice as great as those calculated by the formula. A method, developed from analytical discussion of the phenomenon, is given for adjusting the potentials of the bridge to their correct values.

7. This adjustment of the potentials having been accomplished, the substitution method gives accurate results with resistances at least as high as Io ooo ohms, the limit to its applicability being set only by the difficulty of preparing a suitable standard of reference.

8. The errors due to earth capacities in the case of resistance coils in series is discussed, together with the relative advantages of shielded and unshielded coils.

9. Two methods are given for the measurements of the effective inductances of coils in those cases where it is impracticable to construct primary' standards. The results obtained by the use of these methods are not, however, as precise as those obtained by the method of substitution. In the case of coils having large capacity, where the phase angle is largely dependent on the temperature and other external conditions, these methods are sufficient.

WASHINGTON, September I, I9I I. 